\title{
Bottomonium states in hot asymmetric strange hadronic matter
}

\author{
Amruta Mishra* and Divakar Pathak $\dagger$ \\ Department of Physics, Indian Institute of Technology, \\ Delhi, Hauz Khas, New Delhi - 110 016, India
}

\begin{abstract}
We calculate the in-medium masses of the bottomonium states $(\Upsilon(1 S), \Upsilon(2 S), \Upsilon(3 S)$ and $\Upsilon(4 S))$ in isospin asymmetric strange hadronic matter at finite temperatures. The medium modifications of the masses arise due to the interaction of these heavy quarkonium states with the gluon condensates of QCD. The gluon condensates in the hot hadronic matter are computed from the medium modification of a scalar dilaton field within a chiral SU(3) model, introduced in the hadronic model to incorporate the broken scale invariance of QCD. There is seen to be drop in the masses of the bottomonium states and mass shifts are observed to be quite considerable at high densities for the excited states. The effects of density, isospin asymmetry, strangeness as well as temperature of the medium on the masses of the $\Upsilon$-states are investigated. The effects of the isospin asymmetry as well as strangeness fraction of the medium are seen to be appreciable at high densities and small temperatures. The density effects are the most dominant medium effects which should have observable consequences in the compressed baryonic matter (CBM) in the heavy ion collision experiments in the future facility at FAIR, GSI. The study of the $\Upsilon$ states will however require access to energies higher than the energy regime planned at CBM experiment. The density effects on the bottomonium masses should also show up in the dilepton spectra at the SPS energies, especially for the excited states for which the mass drop is observed to quite appreciable.
\end{abstract}

PACS numbers: 21.65.Jk; 13.75.Jz; 25.75.-q

*Electronic address: amruta@physics.iitd.ac.in,mishra@th.physik.uni-frankfurt.de

${ }^{\dagger}$ Electronic address: dpdlin@gmail.com 


\section{INTRODUCTION}

The study of properties of hadrons in hot and dense matter is an important topic of contemporary research in strong interaction physics. The medium modifications of the hadrons affect the experimental observables from the strongly interacting matter created in the relativistic heavy ion collision experiments. The in-medium properties of the light vector mesons [1 13$]$ are of relevance for observables like dilepton spectra, whereas modifications of the kaons and antikaons modify the production and propagation of these particles. The modifications of the masses of the charm mesons, $D(\bar{D})$ mesons as well as of the charmonium states in the medium can modify the yield of the open charm mesons and of the charmonium states. A larger drop of the masses of the $D$ mesons as compared to the mass drop of the charmonium states can open decay channels of the excited states of charmonium to $D \bar{D}$ in the medium, which are not accessible in vacuum. This can reduce the production of $J / \psi$ from the decay of these excited charmonium states and thereby lead to $\mathrm{J} / \psi$ suppression in heavy ion collision experiments. In high energy nuclear collisions, $J / \psi$ suppression could also be due to formation of quark gluon plasma [4, 5]. In the presence of quark gluon plasma, the quark antiquark potential is screened leading to decrease in the binding energy of the quarkonium states and when the distance between the quark and antiquark becomes larger than the inverse of the Debye mass, the $Q \bar{Q}$ can no longer exist as a bound state. This can lead to step-wise melting of the quarkonium states from the hot deconfined matter and hence cause suppression of the ground states $J / \psi$ and $\Upsilon(1 S)$ as the feed-down from the excited quarkonium states will no longer be possible at temperatures above the dissociation temperatures of the excited states [6]. At still higher temperatures, the quarkonium ground state can also dissociate due to color screening. The suppression of the heavy quarkonium

states in the deconfined matter [7], can also be due to the processes of gluo-dissociation [8] and inelastic parton scattering [9]. In the former process, the quarkonium state breaks up due to interaction with a hard thermal gluon which changes the color singlet quarkonium state to an unbound color octet $Q \bar{Q}$ pair. In the inelastic parton scattering, the quarkonium state interacts with a parton (quark or gluon) through exchange of gluons, leading to dissociation of the heavy quarkonium. An effective field theory describing the quarkonia systems through potentials, namely pNRQCD (potential nonrelativistic QCD) has been extensively used in 
the literature [10]. Within this framework, two mechanisms contributing to the decay width of the quarkonium were identified as singlet-to-octet thermal breakup and Landau damping. In the leading order, these correspond to gluo-dissociation and dissociation of quarkonium due to inelastic parton scattering [11].

The masses of the $D(\bar{D})$ mesons, which consist of a heavy charm quark (antiquark) and a light antiquark (quark), within the QCD sum rule approach, are modified largely due to their interaction with the light quark condensates in the hadronic medium [12]. On the contrary, the masses of the heavy quarkonium states, e.g., the charmonium states, are modified in the leading order due to their interaction with the gluon condensates in the hadronic medium [13 15]. These gluon condensates can be written in terms of the color electric and color magnetic fields. The modifications of the masses of the charmonium states, due to changes in the gluon condensates in the nuclear medium have been studied in the linear density approximation [13, 14], using the leading order QCD formula [16]. The formula for the charmonium mass shift becomes proportional to $\left\langle\frac{\alpha_{s}}{\pi} \vec{E}^{2}\right\rangle$, similar to the second order Stark effect [13, 14], since the Wilson coefficient for the operator $\left\langle\frac{\alpha_{s}}{\pi} \vec{B}^{2}\right\rangle$ vanishes in the nonrelativistic limit. These studies [13, 14] show the drop of the $J / \psi$ mass at the nuclear matter saturation density to be quite small, whereas the masses of the excited charmonium states, e.g. $\psi(3686)$ and $\psi(3770)$ are observed to have appreciable drop in the nuclear medium. The charmonia masses in hot strange hadronic matter have been studied using the leading order QCD formula, due to changes in the gluon condensates in the hadronic medium calculated in a chiral effective model [17]. The gluon condensate of QCD is simulated by a scalar dilaton field introduced in the effective hadronic model to incorporate scale symmetry breaking of QCD. The medium modifications of the decay widths of the charmonium states to $D \bar{D}$ have been studied arising from the mass shifts of the $D$ mesons in the medium [18] as well as of the charmonium states [17] accounting for the internal quark structure of the charmonium as well as $D(\bar{D})$ mesons within the so called ${ }^{3} P_{0}$ model. The charmonium decay widths in the medium have also been studied recently using a field theoretical model for composite hadrons with constituent quarks [19]. Within the QCD sum rule approach, the mass modifications of the charmonium states, $J / \psi$ as well as $\eta_{c}$ have also been studied [20] as arising from the medium modifications of the scalar gluon condensate and the twist-2 
gluon operator, computed from the medium modification of the dilaton field in the chiral effective model. The mass shift of the D meson at finite density was studied using the QCD sum rule approach [12] using condensates upto dimension 4 in the operator product expansion and the dominant contribution to the D meson mass drop comes from the term $m_{c}\langle\bar{q} q\rangle$, which turns out to be appreciable due to the large value of $m_{c}$. The mass shifts and the splitting of the $D-\bar{D}$ (as well as $B-\bar{B}$ ) meson masses have been studied within the QCD sum rule approach by considering condensate operators upto dimension 5 in the operator product expansion. This includes also the contributions from the gluon condensates as well as the mixed quark-gluon condensate [21, 22], with the dominant contribution to the mass modifications of the $D(B)$ mesons arising from the term $m_{c}\langle\bar{q} q\rangle\left(m_{b}\langle\bar{q} q\rangle\right)$ in the operator product expansion. In the recent past, the discovery of many hadrons including the heavy charm [23] and bottom quarks, as well as, the observed suppression of the heavy quarkonia at SPS, RHIC and LHC, which could indicate the formation of deconfined matter, has added further motivation to study the properties of the heavy quarkonium states in the hot and dense matter resulting from heavy ion collision experiments. The charmonium suppression has been observed at SPS energies [24] and at RHIC [25]. The bottomonium suppression has been observed at RHIC for $\mathrm{Au}+\mathrm{Au}$ collision at $\sqrt{s}=200 \mathrm{GeV}$ [26] as well as at $\mathrm{LHC}$ in $\mathrm{Pb}+\mathrm{Pb}$ collision at $\sqrt{s}=2.76 \mathrm{TeV}[27]$. The quarkonia production have been studied using a rate equation [28] for SPS (for Pb-Pb collision at $158 \mathrm{AGeV}(\sqrt{s}=17.3 \mathrm{GeV})$ ), RHIC ( $\mathrm{Au}+\mathrm{Au}$ collisions at $\sqrt{s}=200 \mathrm{GeV})$ and $\mathrm{LHC}(\mathrm{Pb}+\mathrm{Pb}$ collision at $\sqrt{s}=2.76 \mathrm{TeV})$ energies, including their production from initial nucleon nucleon hard scattering and their regeneration from produced QGP, as well as accounting for the in-medium effects of quarkonia [29]. At RHIC and LHC, the medium effects on the quarkonia production are due to the thermal effects, whereas the density effects on the heavy quarkonia production should be important at SPS, CERN as well as at the Compressed baryonic matter (CBM) experiment at FAIR project at the future facility at GSI [30]. At the CBM experiment at FAIR, one of the areas of focus for research will be the study of rare probes, e.g., the heavy quarkonia. The measurements of the production of the heavy quarkonia will be possible due to the high beam intensities and long running times to be used in these experiments. The experimental facility will be using heavy ion beams in fixed target mode with beam energy of about 10 to $45 \mathrm{AGeV}$ ( $\sqrt{s}=4.5$ 
to $10 \mathrm{GeV}$ ). These energies will make the study of charmonia possible quite extensively, whereas, a study of bottomonia production will require access to higher energies, as the top energy of CBM is about the threshold energy when $b$ and $\bar{b}$ can be pair produced which can later combine to form the bottomonium state. The density effects, which seem to the important medium effects on the bottomonia masses in the present investigation, should also be possible to study at SPS energies, where the yields of the $\Upsilon$ states have already been measured in pA collisions at incident energy of $450 \mathrm{AGeV}(\sqrt{s}=29.1 \mathrm{GeV})$ by the NA50 Collaboration [31]. These measurements can provide a baseline for the $\Upsilon$ production studies to be carried out in the ion-ion collisions at higher centre of mass energies.

In the present work, we study the in-medium masses of the bottomonium states, $\Upsilon(1 S)$, $\Upsilon(2 S), \Upsilon(3 S)$ and $\Upsilon(4 S)$ in hot asymmetric strange hadronic matter due to the interaction with the gluon condensates using the leading order QCD formula. The medium modification of the gluon condensate in the hadronic medium is calculated from the medium modification of a scalar dilaton field introduced within a chiral $\mathrm{SU}(3)$ model [32] to incorporate scale symmetry breaking of QCD. The model has been used to study the in-medium properties of the vector mesons [33, 34], kaons and antikaons [35, 36]. The model has then been generalized to chiral $\mathrm{SU}(4)$ to derive the interactions of the charm $D(\bar{D})$ mesons with the light hadron sector and study the effects of isospin asymmetry [37], strangeness [17] and temperature 38] on the mass modifications of these mesons in the hadronic medium. The mass shifts of the charmonium states have been calculated due to the changes in the gluon condensates in the medium [17, 20] obtained from the change of a scalar dilaton field, which mimics the scale symmetry breaking of QCD, in the chiral effective model. In the present investigation, we study the in-medium masses of bottomonium states, obtained from the dilaton field, $\chi$, calculated for the asymmetric strange hadronic matter at finite temperatures.

The outline of the paper is as follows : In section II, we discuss briefly the chiral $S U(3)$ model which has been used to investigate the mass modification of the bottomonium states in the present work. The medium modifications of the bottomonium masses arise from the medium modification of a scalar dilaton field introduced in the hadronic model to incorporate broken scale invariance of QCD leading to QCD trace anomaly. In section III, we summarize the results obtained in the present investigation. 


\section{THE HADRONIC CHIRAL $S U(3) \times S U(3)$ MODEL}

We use a chiral $S U(3)$ model [32, 39 41], which incorporates the scale symmetry breaking of QCD through introduction of a scalar dilaton field [42, 43] within the hadronic model. The modification of the gluon condensates in the hadronic matter is obtained from the medium modification of the scalar dilaton field, which then is used to investigate the in-medium masses of the bottomonium states in the hot isospin asymmetric strange hadronic matter. The effective hadronic chiral Lagrangian density contains the following terms:

$$
\mathcal{L}=\mathcal{L}_{\text {kin }}+\sum_{W=X, Y, V, A, u} \mathcal{L}_{B W}+\mathcal{L}_{\text {vec }}+\mathcal{L}_{0}+\mathcal{L}_{\text {scalebreak }}+\mathcal{L}_{S B}
$$

In Eq. (11), $\mathcal{L}_{\text {kin }}$ is kinetic energy term, $\mathcal{L}_{B W}$ is the baryon-meson interaction term in which the baryon-spin-0 meson interaction term generates the vacuum baryon masses. $\mathcal{L}_{v e c}$ describes the dynamical mass generation of the vector mesons via couplings to the scalar mesons and contain additionally quartic self-interactions of the vector fields. $\mathcal{L}_{0}$ contains the meson-meson interaction terms inducing the spontaneous breaking of chiral symmerty, $\mathcal{L}_{\text {scalebreak }}$ is the scale invariance breaking logarithmic term. $\mathcal{L}_{S B}$ describes the explicit chiral symmetry breaking. To study the hadron properties at finite temperature and densities in the present investigation, we use the mean field approximation, where all the meson fields are treated as classical fields. In this approximation, only the scalar and the vector fields contribute to the baryon-meson interaction, $\mathcal{L}_{B W}$ since for all the other mesons, the expectation values are zero. The scale breaking term [42, 43] of the Lagrangian density

$$
\mathcal{L}_{\text {scalebreak }}=-\frac{1}{4} \chi^{4} \ln \frac{\chi^{4}}{\chi_{0}^{4}}+\frac{d}{3} \chi^{4} \ln \left(\frac{\left(\sigma^{2}-\delta^{2}\right) \zeta}{\sigma_{0}^{2} \zeta_{0}}\left(\frac{\chi}{\chi_{0}}\right)^{3}\right),
$$

where $\chi, \sigma, \zeta$ and $\delta$ are the scalar dilaton field, non-strange scalar field, strange scalar field and the scalar-isovector field respectively. The effect of these logarithmic terms is to break the scale invariance, which leads to the trace of the energy momentum tensor as [44]

$$
\theta_{\mu}^{\mu}=\chi \frac{\partial \mathcal{L}}{\partial \chi}-4 \mathcal{L}=-(1-d) \chi^{4}
$$

The trace of the energy momentum tensor in QCD is given as

$$
\theta_{\mu}^{\mu}=\left\langle\frac{\beta_{Q C D}}{2 g} G_{\mu \nu}^{a} G^{\mu \nu a}\right\rangle+\sum_{i} m_{i} \bar{q}_{i} q_{i}
$$


where the second term in the trace accounts for the finite quark masses, with $m_{i}$ as the current quark mass for the quark of flavor, $i=u, d, s$. The one loop QCD $\beta$ function is given as

$$
\beta_{\mathrm{QCD}}(g)=-\frac{11 N_{c} g^{3}}{48 \pi^{2}}\left(1-\frac{2 N_{f}}{11 N_{c}}\right),
$$

where $N_{c}=3$ is the number of colors and $N_{f}$ is the number of quark flavors. Comparing the trace of the energy momentum tensor in the chiral effective model given by (3) to that of QCD given by (4) and using equation (15), we obtain the scalar gluon condensate related to the dilaton field as,

$$
\left\langle\frac{\alpha_{s}}{\pi} G_{\mu \nu}^{a} G^{\mu \nu a}\right\rangle=\frac{24}{\left(33-2 N_{f}\right)}\left[(1-d) \chi^{4}+\sum_{i} m_{i} \bar{q}_{i} q_{i}\right] .
$$

The second term, $\sum_{i} m_{i} \bar{q}_{i} q_{i}$ can be identified to be the negative of the explicit chiral symmetry breaking term $\mathcal{L}_{S B}$ of equation (11) [20] and is given as

$$
\sum_{i} m_{i} \bar{q}_{i} q_{i}=-\mathcal{L}_{S B}=\left[m_{\pi}^{2} f_{\pi} \sigma+\left(\sqrt{2} m_{k}^{2} f_{k}-\frac{1}{\sqrt{2}} m_{\pi}^{2} f_{\pi}\right) \zeta\right]
$$

We thus see from the equation ([6]) that the scalar gluon condensate $\left\langle\frac{\alpha_{s}}{\pi} G_{\mu \nu}^{a} G^{\mu \nu a}\right\rangle$ is proportional to the fourth power of the dilaton field, $\chi$, in the limiting situation of massless quarks [17].

The coupled equations of motion for the non-strange scalar field $\sigma$, strange scalar field $\zeta$, scalar-isovector field $\delta$ and dilaton field $\chi$, are derived from the Lagrangian density. These equations of motion are solved to obtain the density and temperature dependent values of the scalar fields $(\sigma, \zeta$ and $\delta$ ) and the dilaton field, $\chi$, in the isospin asymmetric hot strange hadronic medium. The values of the scalar fields and the dilaton field, for baryon density, $\rho_{B}$ and temperature, $\mathrm{T}$, are calculated for given values of the strangeness fraction of the medium, $f_{s}=\frac{\sum_{i} s_{i} \rho_{i}}{\rho_{B}}$, and isospin asymmetry parameter, $\eta=-\frac{\sum_{i} I_{3 i} \rho_{i}}{\rho_{B}}$, where $s_{i}$ is the number of strange quarks of baryon $i\left(\mathrm{i}=\mathrm{p}, \mathrm{n}, \Lambda, \Sigma^{ \pm, 0}, \Xi^{-, 0}\right)$ and $I_{3 i}$ is the third component of isospin of the $i$-th baryon. The in-medium masses of heavy quarkonium states are modified due to the modifications of the scalar gluon and the twist-2 gluon condensates [12, 13] in the hadronic medium. These gluon condensates can be written in terms of the color electric and color magnetic fields, $\left\langle\frac{\alpha_{s}}{\pi} \vec{E}^{2}\right\rangle$ and $\left\langle\frac{\alpha_{s}}{\pi} \vec{B}^{2}\right\rangle$ [45] and as has already been mentioned, for heavy quarkonium states, the contribution from the magnetic field part vanishes. Hence the 
mass shift for these states in the hadronic medium arise due to the change in the electric field part, $\frac{\alpha_{s}}{\pi}\left\langle\vec{E}^{2}\right\rangle$, similar to the second order Stark effect [13]. In the leading order mass shift formula derived in the large bottom quark mass limit [16], the shift in the mass of the bottomonium state is given as [13]

$$
\Delta m_{\Upsilon}=-\frac{1}{9} \int d k^{2}\left|\frac{\partial \psi(k)}{\partial k}\right|^{2} \frac{k}{k^{2} / m_{b}+\epsilon}\left(\left\langle\frac{\alpha_{s}}{\pi} E^{2}\right\rangle-\left\langle\frac{\alpha_{s}}{\pi} E^{2}\right\rangle_{0}\right) .
$$

In the above, $m_{b}$ is the mass of the bottom quark, $m_{\Upsilon}$ is the vacuum mass of the bottomonium state and $\epsilon=2 m_{b}-m_{\Upsilon}$ represents the binding energy of the bottomonium state. $\psi(k)$ is the wave function of the bottomonium state in the momentum space, normalized as $\int \frac{d^{3} k}{(2 \pi)^{3}}|\psi(k)|^{2}=1[46]$. It may be noted here that the leading order perturbative formula (8) for the quarkonium $(Q \bar{Q})$ state has been derived [16] in the limit of large quark mass, so that the inverse of the radius of the quarkonium state is much larger than its binding energy, $\epsilon$. Furthermore, the $Q \bar{Q}$ binding in the heavy quarkonium state is approximated to be one-gluon exchange Coulomb potential, where the gluon energy is small compared to the binding energy of the quarkonium state. The wave function of the quarkonium state in this approximation is Coulombic. However, the Coulombic wave function for the quarkonium state may not be realistic for the excited states of the quarkonium state [13]. The mass modifications of the charmonium states due to their interaction with the gluon condensates were studied in Ref. [13, 17], assuming the wave functions to be harmonic oscillator type. In the present work, we also use the harmonic oscillator wave functions to study of the medium modifications of the masses of the bottomonium states, due to their interactions with the gluon condensates.

In the non-relativistic limit, due to vanishing of the contribution from the magnetic field, the expectation value of the scalar gluon condensate can be expressed in terms of the color electric field and hence the mass shift formula for the bottomonium states can be written in terms of the difference in the value of the scalar gluon condensate in the medium and in the vacuum as [17]

$$
\Delta m_{\Upsilon}=\frac{1}{18} \int d k^{2}\left|\frac{\partial \psi(k)}{\partial k}\right|^{2} \frac{k}{k^{2} / m_{b}+\epsilon}\left(\left\langle\frac{\alpha_{s}}{\pi} G_{\mu \nu}^{a} G^{\mu \nu a}\right\rangle-\left\langle\frac{\alpha_{s}}{\pi} G_{\mu \nu}^{a} G^{\mu \nu a}\right\rangle_{0}\right) .
$$

In the present investigation, the wave functions for the bottomonium states are taken to 
be Gaussian and are given as [18]

$$
\psi_{N, l}=\tilde{\mathrm{N}} Y_{l}^{m}(\theta, \phi)\left(\beta^{2} r^{2}\right)^{\frac{l}{2}} e^{-\frac{1}{2} \beta^{2} r^{2}} L_{N-1}^{l+\frac{1}{2}}\left(\beta^{2} r^{2}\right)
$$

where $\beta^{2}=M \omega / \hbar$ is the parameter describing the strength of the harmonic oscillator potential, $M=m_{b} / 2$ is the reduced mass of the bottom quark and bottom anti-quark system, $L_{p}^{k}(z)$ is the associated Laguerre polynomial. $\tilde{\mathrm{N}}$ is the normalization constant determined from $\int\left|\psi_{N, l}(\mathbf{r})\right|^{2} d^{3} r=1$. The oscillator constant $\beta$ is calculated from the decay widths of the bottomonium state to $e^{+} e^{-}$given by the formula [47, 48]

$$
\Gamma_{\Upsilon \rightarrow e^{+} e^{-}}=\frac{16 \pi \alpha^{2}}{9 M_{\Upsilon}^{2}}|\psi(\mathbf{0})|^{2},
$$

where, $\alpha=\frac{1}{137}$ is the fine structure constant and $\psi(\mathbf{0})$ is the wave function of the bottomonium state at the origin. Knowing the wave functions of the bottomonium states and calculating the medium modification of the scalar gluon condensate from the in-medium dilaton field and the scalar fields, we obtain the mass shift of the bottomonium states. In the next section we discuss the results for these in-medium bottomonium masses in hot asymmetric strange hadronic matter obtained in the present work.

\section{RESULTS AND DISCUSSIONS}

In this section, we investigate the effects of density and temperature on the masses of the bottomonium states for given isospin-asymmetry and strangeness of the hadronic medium. Within a chiral SU(3) model, the scalar gluon condensate in the hadronic matter has been calculated from the change in the dilaton field and scalar fields. Using the leading order QCD formula for the mass shift of heavy quarkonium state, the medium modification of the charmonium states $J / \psi, \psi(3686)$ and $\psi(3770)$ were studied from the modification of the gluon condensate in the hadronic matter [13, 17]. In the present work, we investigate the medium modification of the $\Upsilon$-states using equation (9) from the value of the scalar gluon condensate calculated in the chiral SU(3) model. The value of the mass of the b-quark is taken to be $m_{b}=5.36 \mathrm{GeV}$ [49] in the present investigation. This value of $m_{b}$ is similar to the value of $5.1 \mathrm{GeV}$ [50] and $5.005 \mathrm{GeV}$ [48] used in the potential model calculations for the study of the bottomonium spectroscopy. As has already been mentioned the wave 

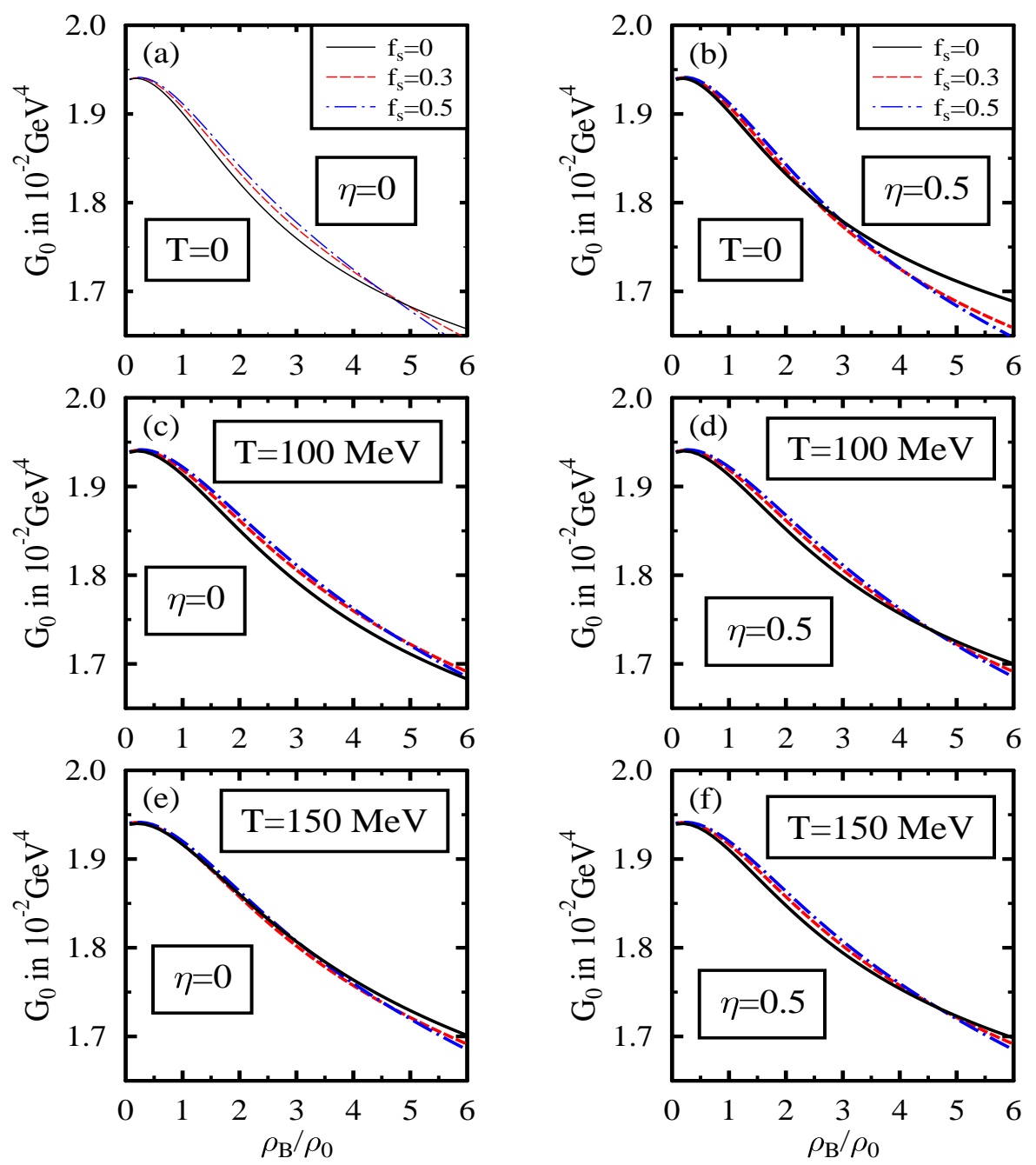

FIG. 1: (Color online) The scalar gluon condensate, $G_{0} \equiv\left\langle\left(\alpha_{s} / \pi\right) G_{\mu \nu}^{a} G^{\mu \nu a}\right\rangle$ is plotted as a function of the baryon density, $\rho_{B}$ in units of nuclear matter saturation density, $\rho_{0}$. This is shown for different values of the temperature for typical values of the strangeness fraction of the medium, $f_{s}$, in subplots (a), (c) and (e) for the isospin symmetric matter and in subplots (b), (d) and (f) for the isospin asymmetric matter with $\eta=0.5$. 

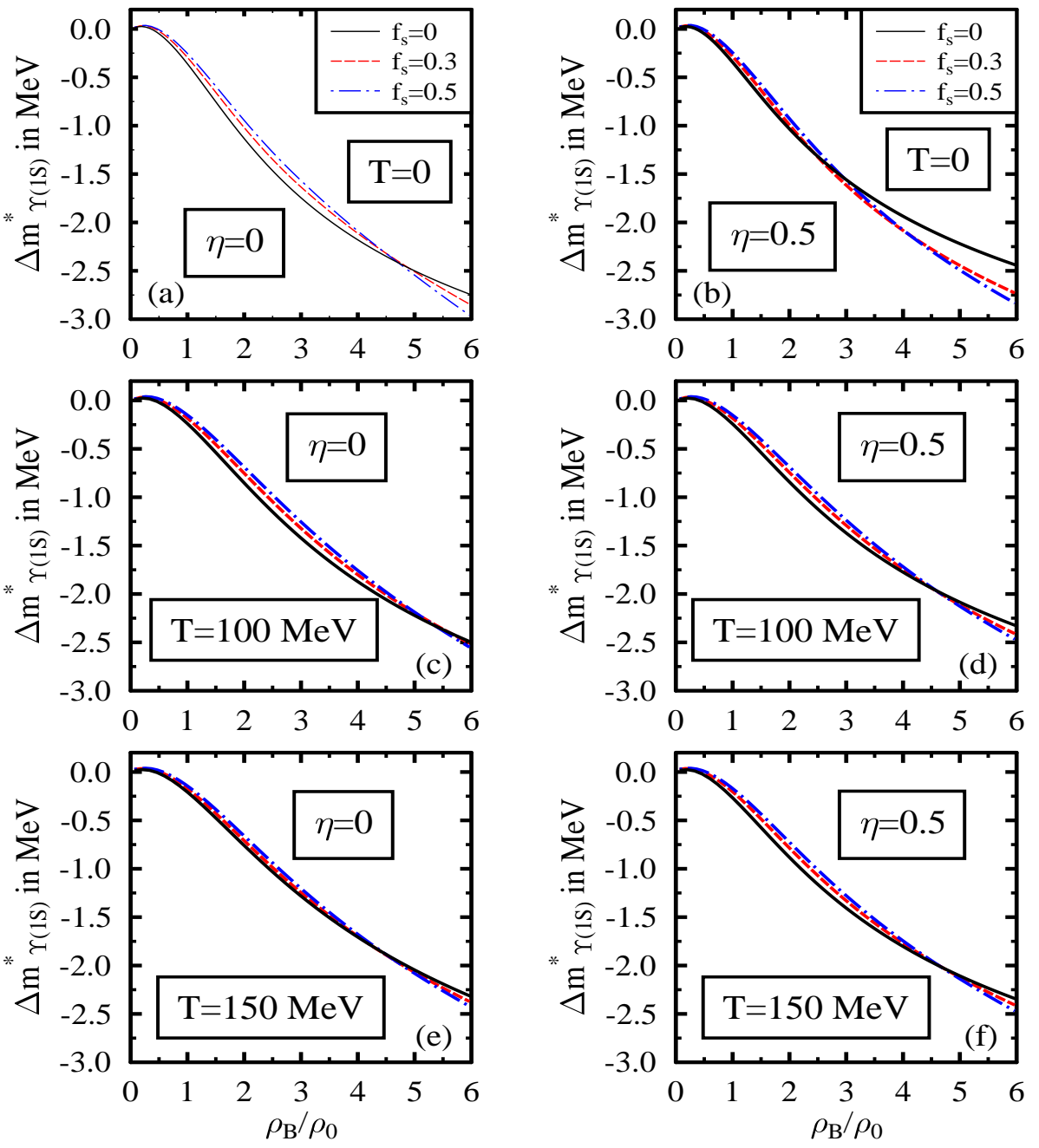

FIG. 2: (Color online) The mass shift of $\Upsilon(1 S)$ plotted as a function of the baryon density in units of nuclear matter saturation density at given temperatures, for different values of the strangeness fraction for the isospin symmetric $(\eta=0)$ as well as isospin asymmetric $(\eta=0.5)$ hadronic matter.

functions of the $\Upsilon$-states have been taken to be harmonic oscillator eigenfunctions as given by equation (10) and the values of the harmonic oscillator strength, $\beta$ for the states are determined from the experimental values of the leptonic decay widths of the bottomonium states, given by equation (11). The values of the decay widths $\left(\Upsilon \rightarrow e^{+} e^{-}\right)$of $1.34 \mathrm{keV}$, 

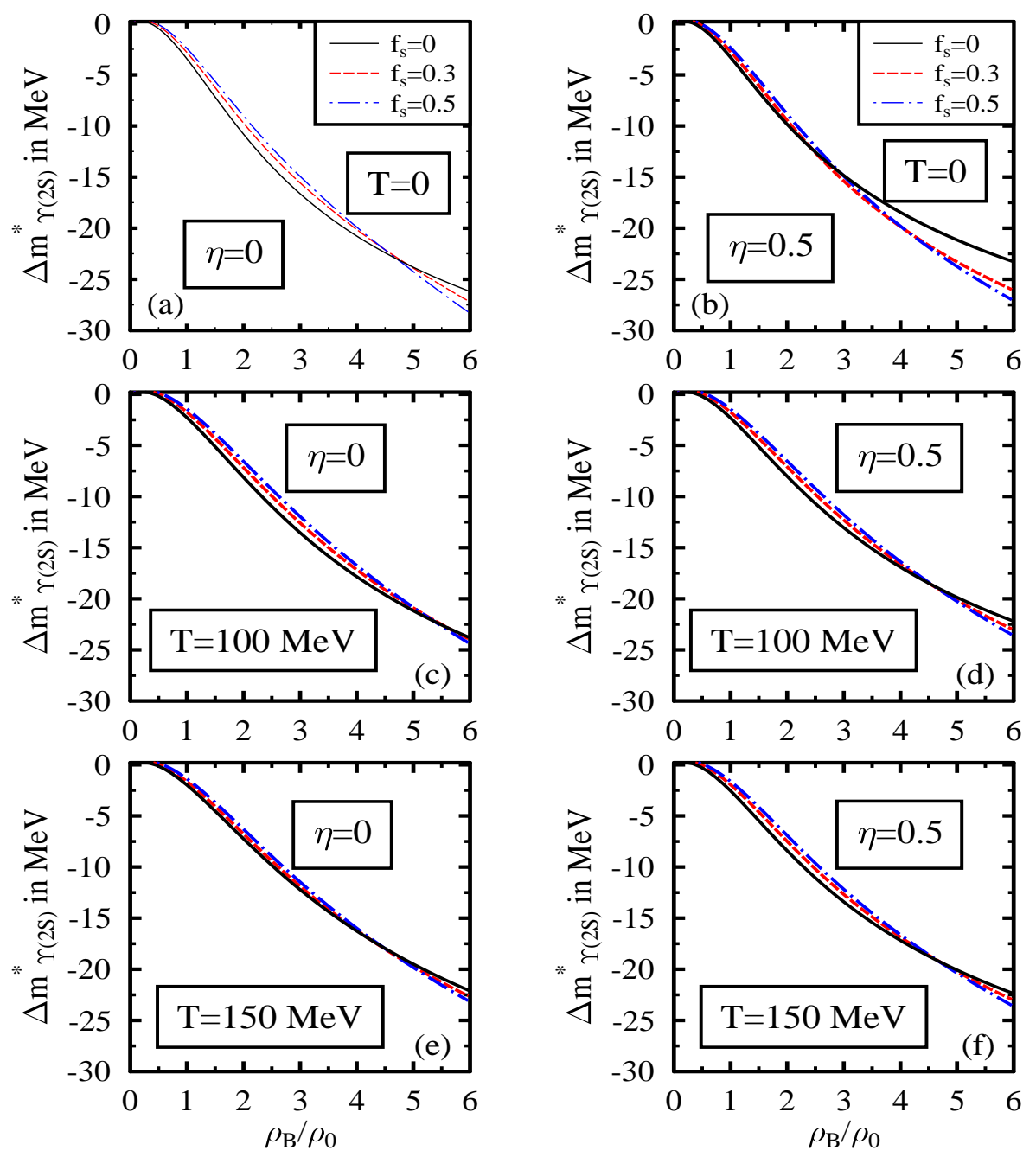

FIG. 3: (Color online) The mass shift of $\Upsilon(2 S)$ plotted as a function of the baryon density in units of nuclear matter saturation density at given temperatures, for different values of the strangeness fraction for the isospin symmetric $(\eta=0)$ as well as isospin asymmetric $(\eta=0.5)$ hadronic matter.

$0.612 \mathrm{keV}, 0.443 \mathrm{keV}$ and $0.272 \mathrm{keV}$ for the $\Upsilon$-states, $\Upsilon(1 S), \Upsilon(2 S), \Upsilon(3 S)$ and $\Upsilon(4 S)$ [51] give the values of the harmonic oscillation strength, $\beta$ as 1309.2, 915.4, 779.75 and 638.6 $\mathrm{MeV}$ respectively. These values of the parameter $\beta$ may be compared with the calculations of the heavy quarkonium systems using a quark model [48] with a linear confining potential, 

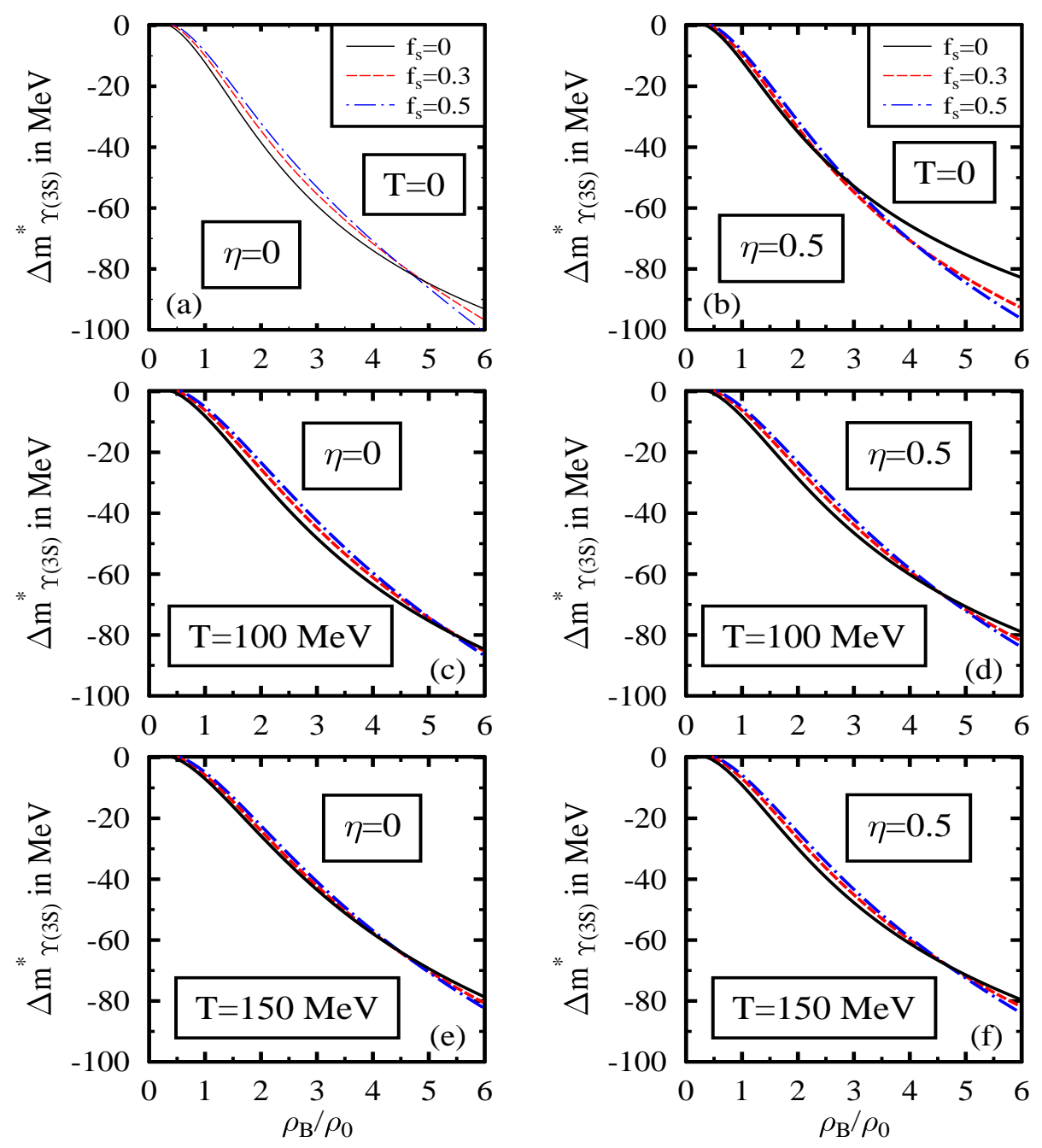

FIG. 4: (Color online) The mass shift of $\Upsilon(3 S)$ plotted as a function of the baryon density in units of nuclear matter saturation density at given temperatures, for different values of the strangeness fraction for the isospin symmetric $(\eta=0)$ as well as isospin asymmetric $(\eta=0.5)$ hadronic matter.

calculated as 1291 and $953.46 \mathrm{MeV}$ for the $\Upsilon(1 S)$ and $\Upsilon(2 S)$ states.

In figure 1, the density dependence of the scalar gluon condensate, $G_{0}=\left\langle\left(\alpha_{s} / \pi\right) G_{\mu \nu}^{a} G^{\mu \nu a}\right\rangle$ is shown for temperatures, $\mathrm{T}=0,100$ and $150 \mathrm{MeV}$, for the isospin symmetric matter in subplots (a), (c) and (e), for values of the strangeness fraction, $f_{s}$ as $0,0.3$ and 0.5 . These 

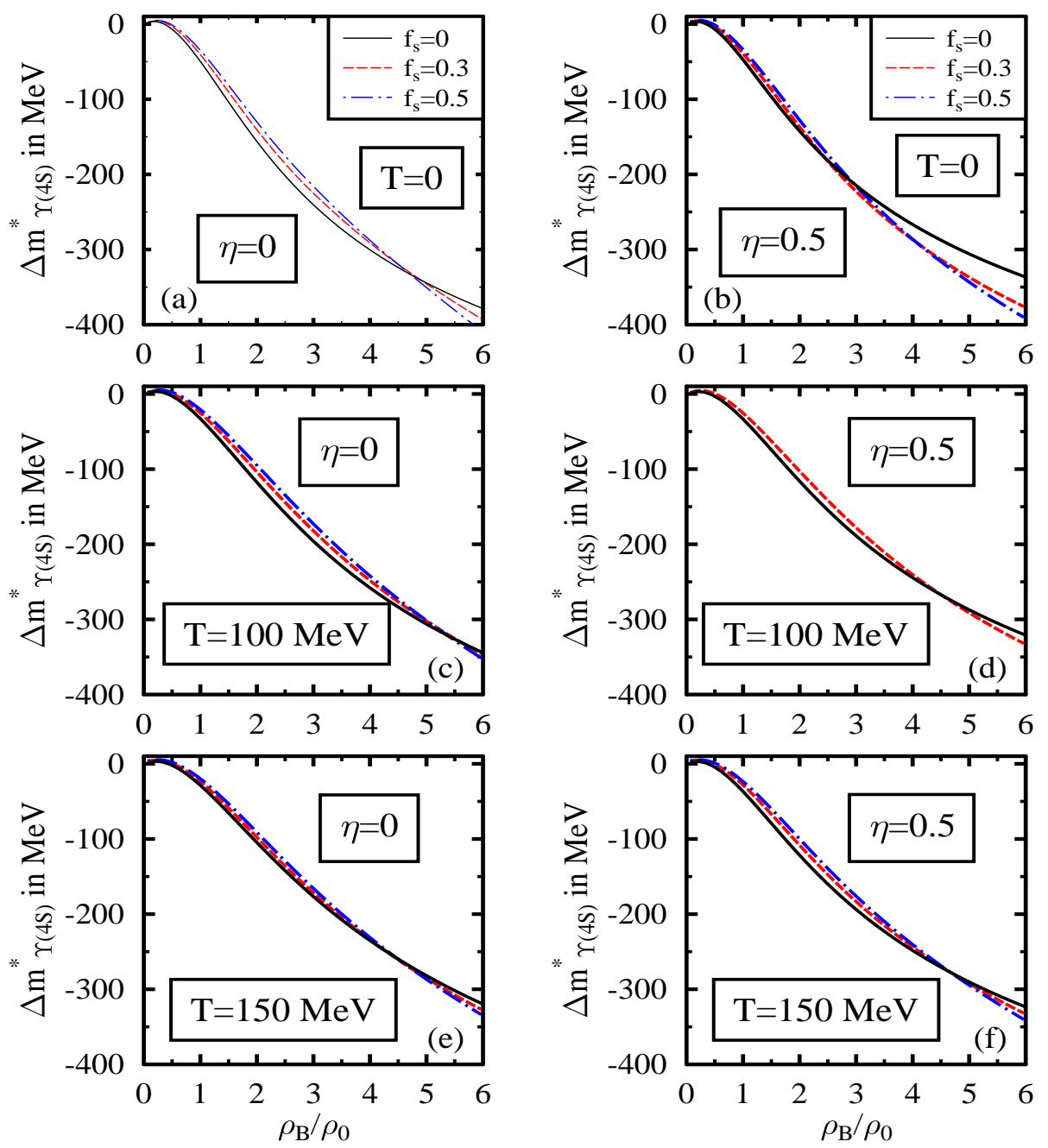

FIG. 5: (Color online) The mass shift of $\Upsilon(4 S)$ plotted as a function of the baryon density in units of nuclear matter saturation density at given temperatures, for different values of the strangeness fraction for the isospin symmetric $(\eta=0)$ as well as isospin asymmetric $(\eta=0.5)$ hadronic matter.

are compared with the values for the isospin asymmetric matter with $\eta=0.5$ plotted in (b), (d) and (f). The scalar gluon condensate is calculated in the chiral SU(3) model, using equations (6) and (7), from the in-medium values of the dilaton field, $\chi$ and the scalar fields, $\sigma, \zeta$ and $\delta$ in the isospin asymmetric strange hadronic medium, obtained by 

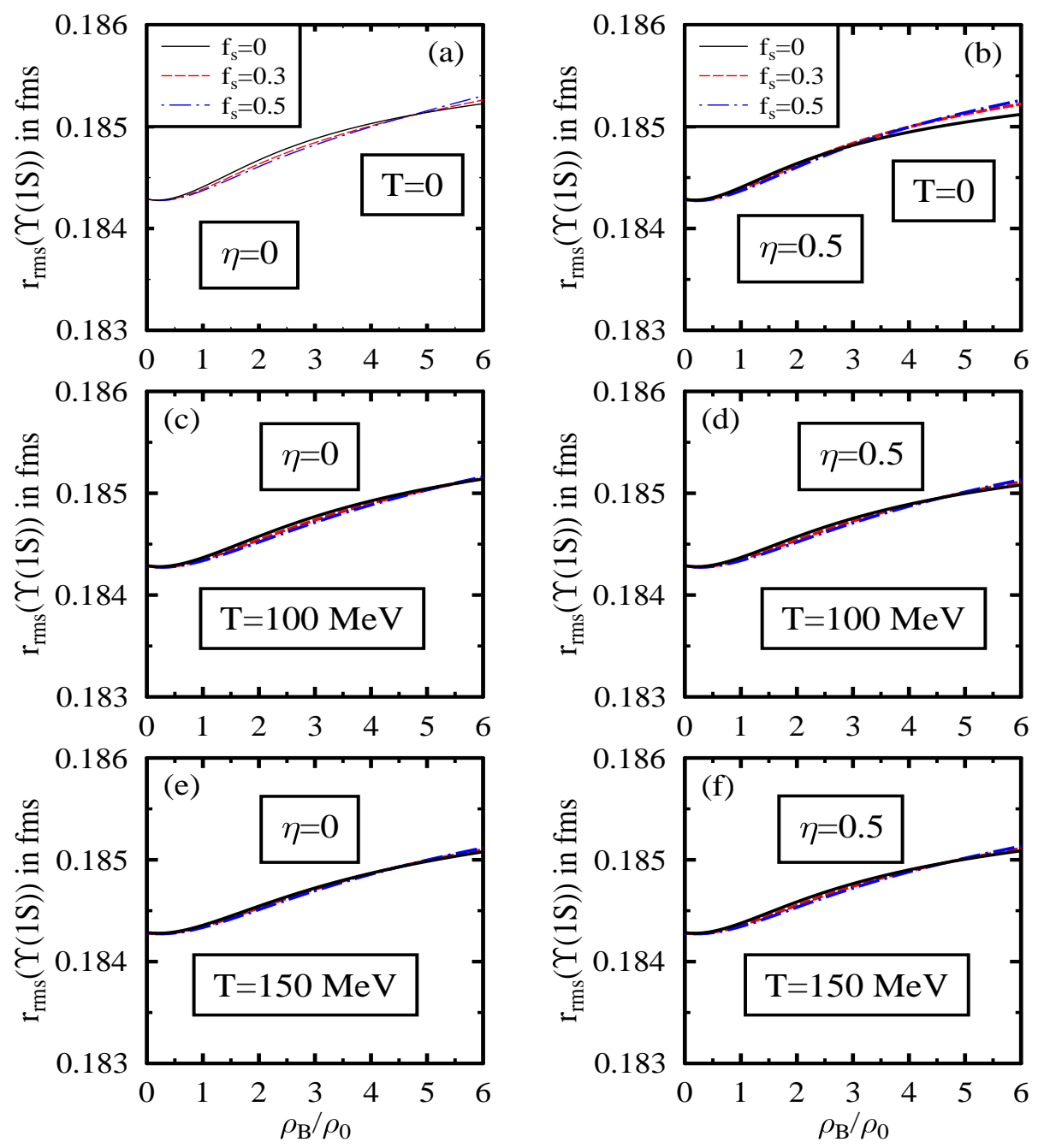

FIG. 6: (Color online) The rms radius of $\Upsilon(1 S)$ plotted as a function of the baryon density in units of nuclear matter saturation density at given temperatures, for different values of the strangeness fraction for the isospin symmetric $(\eta=0)$ as well as isospin asymmetric $(\eta=0.5)$ hadronic matter.

solving the coupled equations of motion of these fields. For the isospin symmetric nuclear matter, the value of the scalar gluon condensate changes from the vacuum value of $1.9387 \times 10^{-2} \mathrm{GeV}^{4}$ to $1.9 \times 10^{-2} \mathrm{GeV}^{4}$ at the nuclear matter saturation density, and to $1.822 \times 10^{-2} \mathrm{GeV}^{4}$ and $1.7155 \times 10^{-2} \mathrm{GeV}^{4}$ at densities $2 \rho_{0}$ and $4 \rho_{0}$ respectively. In the pres- 

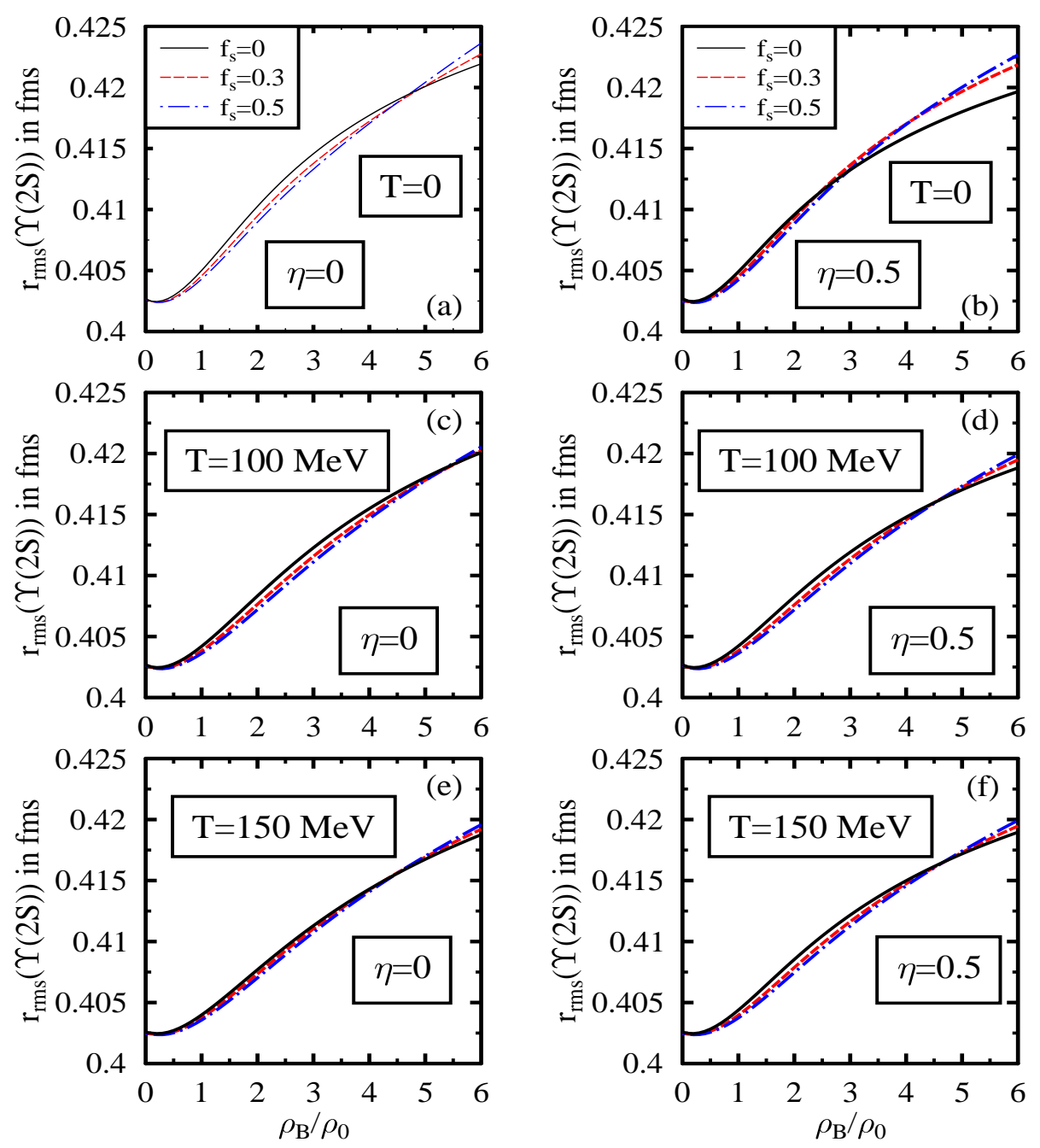

FIG. 7: (Color online) The rms radius of $\Upsilon(2 S)$ plotted as a function of the baryon density in units of nuclear matter saturation density at given temperatures, for different values of the strangeness fraction for the isospin symmetric $(\eta=0)$ as well as isospin asymmetric $(\eta=0.5)$ hadronic matter.

ence of hyperons in the system, the dilaton field is observed to have a larger drop in the medium. When the quark masses are neglected, the second term of the trace of the energy momentum tensor does not contribute and the scalar gluon condensate becomes proportional to the fourth power of the dilaton field. However, the contributions from the quark 

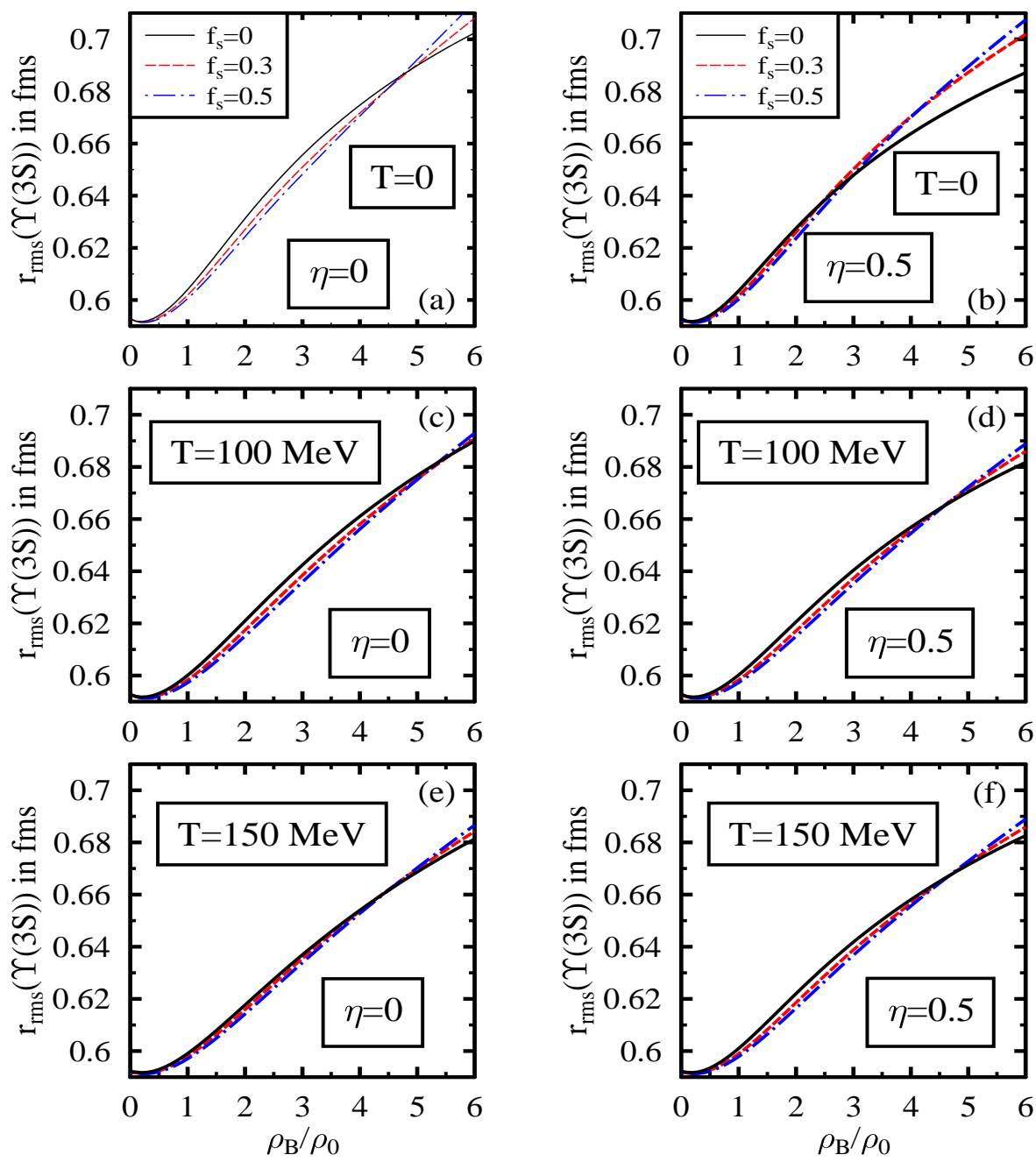

FIG. 8: (Color online) The rms radius of $\Upsilon(3 S)$ plotted as a function of the baryon density in units of nuclear matter saturation density at given temperatures, for different values of the strangeness fraction for the isospin symmetric $(\eta=0)$ as well as isospin asymmetric $(\eta=0.5)$ hadronic matter.

condensate term in the trace of the energy momentum tensor expressed in terms of the scalar fields, $\sigma$ and $\zeta$, modifies the value of the scalar gluon condensate in the medium. At zero temperature, for symmetric hadronic matter, the modification arising from the finite strangeness fraction is observed to be small, with the value of the scalar gluon condensate 

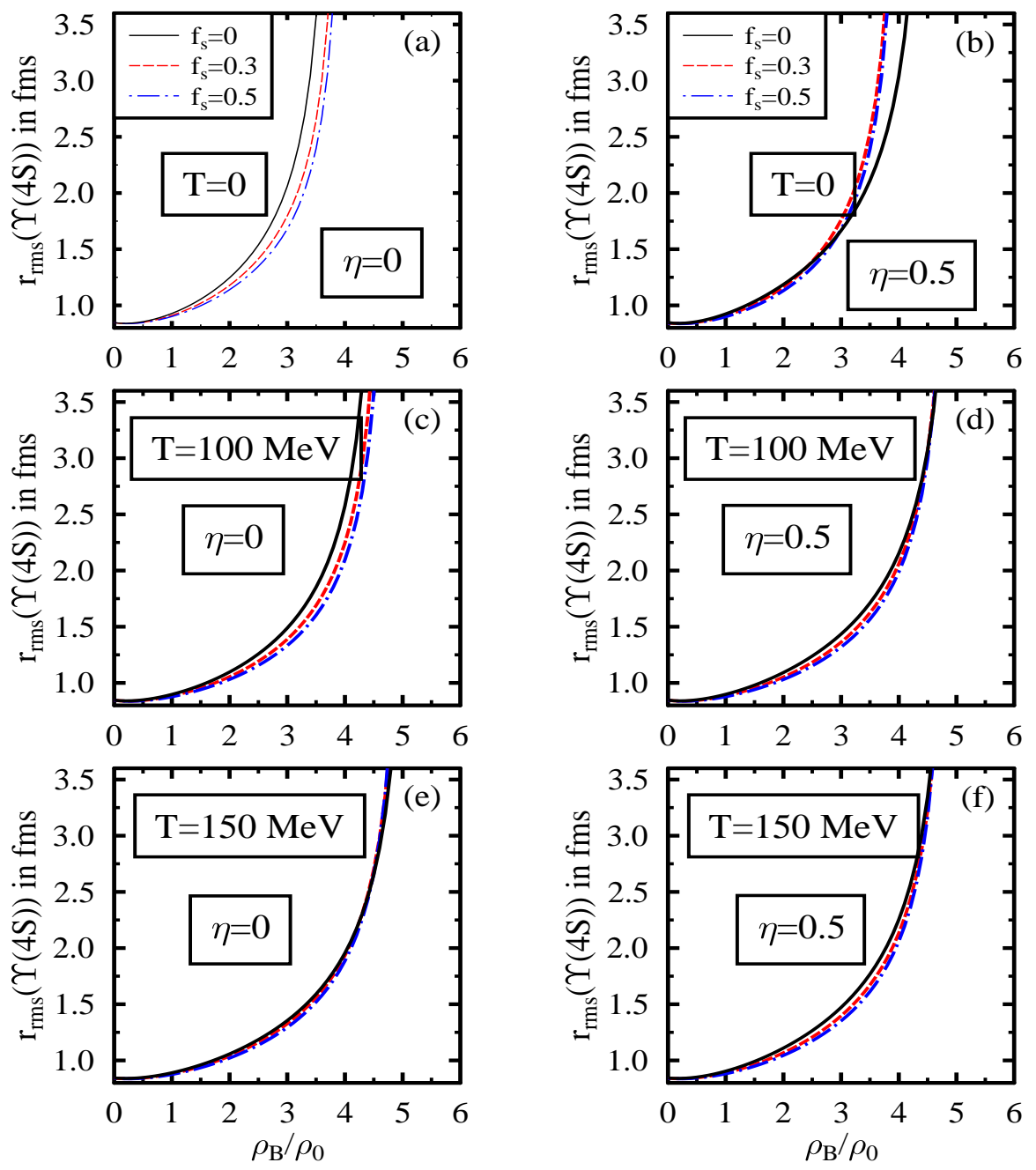

FIG. 9: (Color online) The rms radius of $\Upsilon(4 S)$ plotted as a function of the baryon density in units of nuclear matter saturation density at given temperatures, for different values of the strangeness fraction for the isospin symmetric $(\eta=0)$ as well as isospin asymmetric $(\eta=0.5)$ hadronic matter.

amounting to $1.9075 \times 10^{-2} \mathrm{GeV}^{4}, 1.8336 \times 10^{-2} \mathrm{GeV}^{4}$, and $1.7223 \times 10^{-2} \mathrm{GeV}^{4}$, for $f_{s}=0.3$ and $1.9117 \times 10^{-2} \mathrm{GeV}^{4}, 1.8412 \times 10^{-2} \mathrm{GeV}^{4}$, and $1.7248 \times 10^{-2} \mathrm{GeV}^{4}$, for $f_{s}=0.5$, for densities of $\rho_{0}, 2 \rho_{0}$ and $4 \rho_{0}$ respectively. At $\mathrm{T}=0$, the effect from the finite $f_{s}$ is observed to be larger for the isospin asymmetric case as compared to the symmetric situation. The difference 
in the in-medium gluon condensate from the vacuum value is observed to decrease as the temperature is increased, due to the fact that the drop in the magnitude in the dilaton and the scalar fields, $\sigma$ and $\zeta$ decrease with increase in temperature.

In figures 2, 3, 4 and 5, we show the density dependence of the mass shifts of the upsilon states, $\Upsilon(1 S), \Upsilon(2 S), \Upsilon(3 S)$ and $\Upsilon(4 S)$ respectively, in isospin asymmetric strange hadronic matter. The mass of $\Upsilon(1 S)$ is observed to drop from its vacuum value of $9460.3 \mathrm{MeV}$ to 9459.94 MeV (9458.12 MeV) at density of $\rho_{0}\left(4 \rho_{0}\right)$ for symmetric nuclear matter at zero temperature. For the asymmetric nuclear matter $(\eta=0.5)$, the in-medium mass is changed to $9459.956 \mathrm{MeV}(9458.36 \mathrm{MeV})$ at density $\rho_{0}\left(4 \rho_{0}\right)$. The strangeness fraction in the medium is seen to lead to marginal modification for the symmetric matter, whereas it is larger for the asymmetric situation for $\mathrm{T}=0$. However, the value of the mass shift remains small, of the order of about $3 \mathrm{MeV}$ at a density of six times nuclear matter density. With increase in temperature, the drop is seen to be even less (of the order of $2.5 \mathrm{MeV}$ at a density of $6 \rho_{0}$ ). For $\Upsilon(2 S)$ plotted in figure 3, the mass is observed to be modified to 10019.83 $\mathrm{MeV}(10002.49 \mathrm{MeV})$ at $\rho_{0}\left(4 \rho_{0}\right)$ from its vacuum value of $10023.26 \mathrm{MeV}$. However, the effects from temperature, strangeness and isospin asymmetry remain small as compared to the modification of the mass from density effects. For $\Upsilon(3 S)$ shown in figure 4 , the mass shift is observed to be $12.21 \mathrm{MeV}(73.916 \mathrm{MeV})$ from its vacuum value of 10355.2 $\mathrm{MeV}$, at density of $\rho_{0}\left(4 \rho_{0}\right)$ for symmetric nuclear matter at $\mathrm{T}=0$. The dependence on $f_{s}$ is seen to be larger for the asymmetric case at high densities. The mass shift of the $\Upsilon$ state is proportional to the difference in the value of the scalar gluon condensate from the vacuum value, with the constant of proportionality determined by the integral of equation (9) whose integrand is given in terms of the wave function of the specific $\Upsilon$ state. For given values of the temperature, isospin asymmetry, strangeness fraction and density of the medium, the ratio of the magnitudes of the mass shifts for the $\Upsilon(1 S), \Upsilon(2 S), \Upsilon(3 S)$ and $\Upsilon(4 S)$ states turns out to be the ratio of the magnitudes of the integral calculated from their respective wave functions. These mass shifts are observed to be in the ratio $\Delta m_{\Upsilon(1 s)}: \Delta m_{\Upsilon(2 s)}: \Delta m_{\Upsilon(3 s)}: \Delta m_{\Upsilon(4 s)}=1: 9.53: 33.9: 137.8$. The mass drop of the excited states are thus observed to be larger for the excited states and for $\Upsilon(4 S)$, the drop in the mass is seen to be about $49.64 \mathrm{MeV}(300.4 \mathrm{MeV})$ from the vacuum mass of $10579.4 \mathrm{MeV}$, at 
a density of $\rho_{0}\left(4 \rho_{0}\right)$ for symmetric nuclear matter at zero temperature. We might note here that the mass of the $\Upsilon$ state has initially an increase with density, as the contribution from the finite quark mass term dominates over the first term in the expression for the scalar gluon condensate given by equation (6) . The rise is observed to be about $3.4 \mathrm{MeV}$ at a density of about $0.2 \rho_{0}$ for $\Upsilon(4 S)$, followed by a drop in the mass as the density is further increased. In the case when the finite quark mass term in the trace of the energy momentum tensor in QCD is neglected, there is seen to be a monotonic drop of the $\Upsilon$ states with increase in density, since the dilaton field dereases with density.

With the harmonic oscillator parameter, $\beta$ of the wave function of the bottomonium states as fitted from their leptonic decay widths, the root mean square radii for the states, $\Upsilon(1 S), \Upsilon(2 S), \Upsilon(3 S)$ and $\Upsilon(4 S)$ are obtained as $0.1843,0.4027,0.5928$ and 0.8466 fermis respectively in the present investigation. These values are similar to the values for the rms radii obtained from using a Cornell potential for the bottomonium bound state of Ref. [52], of $0.2,0.48,0.72$ and 0.92 fermis for these bottomonium states and, 0.1869 and 0.3865 fermis for the $\Upsilon(1 S)$ and $\Upsilon(2 S)$ states calculated in a quark model [48] using a confining linear potential. In the hadronic medium, due to the mass drop in the bottomonium states, the strength of the harmonic oscillator wave function, $\beta$ is modified from which we can obtain an estimate for the size of the bottomonium state in the hadronic medium. For the states, $\Upsilon(1 S), \Upsilon(2 S), \Upsilon(3 S)$ and $\Upsilon(4 S)$, the change in the strength of the quarkonium wave function is obtained [13, 17] from $\Delta \beta^{2}=\frac{2}{3} M_{\Upsilon} \Delta M_{\Upsilon}, \Delta \beta^{2}=\frac{2}{7} M_{\Upsilon} \Delta M_{\Upsilon}, \Delta \beta^{2}=\frac{2}{11} M_{\Upsilon} \Delta M_{\Upsilon}$, $\Delta \beta^{2}=\frac{2}{15} M_{\Upsilon} \Delta M_{\Upsilon}$, for the states $\Upsilon(1 S), \Upsilon(2 S), \Upsilon(3 S)$, and $\Upsilon(4 S)$ respectively. The rms radii of these states in the isospin asymmetric hot strange hadronic matter are plotted in figures 6, 7, 8 and 9, There is seen to be increase in the rms radii of these states with density and this rise is seen to be especially prominent for $\Upsilon(4 S)$ state. These can have consequences on the scattering cross-sections of these states by the baryons in the medium. This is because the leading order QCD calculations [16, 53] show that this scattering crosssection is proportional to the root mean square radii, $r_{r m s}^{2}$ [18]. Hence the increase in the size of the $\Upsilon$-states in the medium can enhance their decay widths. 


\section{SUMMARY}

In the present work, we have investigated the mass modification of the bottomonium states $(\Upsilon(1 S), \Upsilon(2 S), \Upsilon(3 S)$ and $\Upsilon(4 S))$, using the leading order QCD mass formula, from the medium modification of the scalar gluon condensate. The gluon condensate in the isospin asymmetric strange hadronic matter is calculated in a chiral SU(3) model. The broken scale invariance of QCD is incorporated into the hadronic model by introducing a scalar dilaton field. In the limit of massless quarks, the scalar gluon condensate is proportional to the fourth power of the dilaton field. However, in the case of finite quark masses, there is contribution to the scalar gluon condensate from the quark condensates, which are determined in the chiral $\mathrm{SU}(3)$ model from the values of the scalar fields, $\sigma, \zeta$ and $\delta$ in the hadronic medium. The mass shifts of the $\Upsilon$-states are observed to be larger for the excited states and are of the order of few hundred $\mathrm{MeV}$ for $\Upsilon(4 S)$ state. The density effect is the dominant medium effect as compared to the effects from strangeness fraction, isospin asymmetry and the temperature of the hadronic matter. These mass shifts can possibly show in the dilepton spectra arising from the compressed baryonic matter in the future facility at GSI, when there is access to higher energies as compared to the planned energy range at CBM. The density effects of the mass modifications of the bottomonium states should also show up in the dilepton spectra at SPS. The $\Upsilon$ states have already been measured in pA collisions at incident energy of $450 \mathrm{AGeV}(\sqrt{s}=29.1 \mathrm{GeV})$ by the NA50 Collaboration [31], which can provide a baseline

for the $\Upsilon$ production studies to be carried out in the ion-ion collisions at higher centre of mass energies at SPS. In the present work, the medium modifications of rms radii of the $\Upsilon$ states have been calculated due to the change in the strength in the harmonic oscillator wave functions of these states in the hadronic medium. There is seen to be an increase in the rms radii of these states with density in the present investigation. This can lead to appreciable contribution to the decay widths of the bottomonium states due to scattering from the nucleons in the hadronic medium. 


\section{Acknowledgments}

AM would like to thank S.P.Misra and H. Mishra for discussions and Department of

Science and Technology, Government of India (project number SR/S2/HEP-031/2010) for financial support. DP acknowledges financial support from University of Grants Commission, India (Sr. No. 2121051124, Ref. No. 19-12/2010(i)EU-IV).

[1] R. Rapp and J. Wambach, Adv. Nucl. Phys. 25 (2000) 1; R. Rapp, G. Chanfray and J. Wambach, Nucl. Phys. A 617 (1997) 472.

[2] T. Hatsuda and S. H. Lee, Phys. Rev. C 46, R34 (1992); T. Hatsuda, S. H. Lee and H. Shiomi, Phys. Rev. C 52, 3364 (1995).

[3] F. Klingl, N. Kaiser, W. Weise, Nucl. Phys. A 624,527 (1997).

[4] J. P. Blaizot and J. Y. Ollitrault, Phys. Rev. Lett. 77, 1703 (1996).

[5] T. Matsui and H. Satz, Phys. Lett. B 178, 416 (1986).

[6] H. Satz, arXiv:1310.1209 (hep-ph).

[7] A. Mocsy, P. Petreczky, M. Strikland, Int. Jour. Mod. Phys. A 28, 1340012 (2013).

[8] X-M. Xu,D. Kharzeev,H.Satz and X-N Wang, Phys. Rev. C 53, 3051, 1996; D. Kharzeev and H. Satz, Phys. Lett. B334, 1994, 155.

[9] L. Grandchamp and R. Rapp, Phys. Lett. B 523 (2001) 60; L. Grandchamp and R. Rapp, Nucl. Phys. A 709 (2002) 415.

[10] N. Brambilla, A. Pineda, J. Soto, A. Vairo, Nucl. Phys. B 566 (2000) 275.

[11] N. Brambilla, M. A. Escobedo, J. Ghiglieri and A. Vairo, JHEP 1112 (2011) 116; N. Brambilla, M. A. Escobedo, J. Ghiglieri and A. Vairo, JHEP 1305 (2013) 130; M.A. Escobedo, arXiv 1401.4892 (hep-ph).

[12] Arata Hayashigaki , Phys. Lett. B 487, 96 (2000).

[13] Su Houng Lee and Che Ming Ko, Phys. Rev. C 67, 038202 (2003).

[14] Su Houng Lee and Che Ming Ko, Prog. Theor. Phys. Suppl. 149, 173, 2003.

[15] Sugsik Kim, Su Houng Lee, Nucl. Phys. A 679, 517 (2001).

[16] M.E. Peskin, Nucl. Phys. B156, 365 (1979). 
[17] Arvind Kumar and Amruta Mishra, Eur. Phys. Jour. A 47, 164 (2011).

[18] Bengt Friman, Su Houng Lee and Taesoo Song Phys. Lett. B 548, 153 (2002).

[19] Amruta Mishra and S. P. Misra, arxiv:1308 0202 (nucl-th).

[20] Arvind Kumar and Amruta Mishra, Phys. Rev. C 82, 045207 (2010).

[21] T. Hilger, R. Thomas and B. Kaempfer, Phys. Rev. C 79, 025202 (2009).

[22] T. Hilger, R. Schulze and B. Kaempfer, J. Phys. G. Nucl. Part. Phy. 37094054 (2010).

[23] M. Nielsen, F. S. Navarra and S. H. Lee, Phys. Rep. 497, 41 (2010).

[24] NA50 collaboration, L. Ramello et al, Nucl. Phys. A 774, 59 (2006).

[25] A. Adare et al (PHENIX collaboration) Phys. Rev. Lett. 98, 232301 (2007).

[26] R. Reed et al (STAR Collaboration) arXiv: 1109.3891 (nucl-ex).

[27] S. Chatrchyan et al (CMS collaboration), Phys. Rev. Lett. 107, 052302 (2011).

[28] R. Rapp, Blaschke and P. Crochet, Prog. Part. Nucl. Phys. 65209 (2010); X. Zhao, A. Emerick and R. Rapp, Nucl. Phys. A 904 611c-614c (2013).

[29] T. Song, K. C. Han and C. M. Ko, Phys. Rev. C85, 014902 (2012); C. M. Ko, K. Han and T. Song, Nucl. Phys. A 910, 474 (2013).

[30] http://www.gsi.de/fair/experiments.

[31] NA50 Collaboration, B. Alessandro et al, Phys. Lett. B635 260 (2006).

[32] P. Papazoglou, D. Zschiesche, S. Schramm, J. Schaffner-Bielich, H. Stöcker, and W. Greiner, Phys. Rev. C 59, 411 (1999).

[33] D. Zschiesche, A. Mishra, S. Schramm, H. Stöcker and W. Greiner, Phys. Rev. C 70, 045202 (2004).

[34] A. Mishra, K. Balazs, D. Zschiesche, S. Schramm, H. Stöcker, and W. Greiner, Phys. Rev. C 69, 024903 (2004).

[35] A. Mishra, E. L. Bratkovskaya, J. Schaffner-Bielich, S. Schramm and H. Stöcker, Phys. Rev. C 70, 044904 (2004), A. Mishra and S. Schramm, Phys. Rev. C 74, 064904 (2006).

[36] Amruta Mishra, Arvind Kumar, Sambuddha Sanyal, S. Schramm, Eur. Phys, J. A 41, 205 (2009).

[37] Amruta Mishra and Arindam Mazumdar, Phys. Rev. C 79, 024908 (2009).

[38] A. Mishra, E. L. Bratkovskaya, J. Schaffner-Bielich, S.Schramm and H. Stöcker, Phys. Rev. 
C 69, 015202 (2004).

[39] S.Weinberg, Phys. Rev. 1661568 (1968).

[40] S. Coleman, J. Wess, B. Zumino, Phys. Rev. 1772239 (1969); C.G. Callan, S. Coleman, J. Wess, B. Zumino, Phys. Rev. 1772247 (1969).

[41] W. A. Bardeen and B. W. Lee, Phys. Rev. 1772389 (1969).

[42] J. Schechter, Phys. Rev. D 21, 3393 (1980).

[43] J.Ellis, Nucl. Phys. B 22, 478 (1970); B. A. Campbell, J. Ellis and K. A. Olive, Nucl. Phys. B 345,57 (1990).

[44] Erik K. Heide, Serge Rudaz and Paul J. Ellis, Nucl. Phys. A 571, (2001) 713.

[45] Xuemin Jin, Thomas D. Cohen, R. J. Furnstahl and David K. Griegel, Phys. Rev. C 45, 1881 (1992).

[46] Su Houng Lee and Kenji Morita, Phys. Rev. D 79, 011501(R) (2009).

[47] R. Van Royen and V. F. Weiskopf, Nuovo Cimento 50, 617 (1967).

[48] S. P. Misra, S. Naik and A. R. Panda, Pramana, J. Phys. 28, 131 (1987).

[49] S. F. Radford and W. W. Repko, Phys. Rev. D 75, 074031 (2007).

[50] G. Bhanot and M. E. Peskin, Nucl. Phys. B 156, 391 (1979).

[51] J. Beringer et al (Particle Data Group), Phys. Rev. D 86, 010001 (2012).

[52] E. Eichten, K. Gottfried, T. Kinoshita, K. D. Lane and T. M. Yan, Phys. Rev. D 21, 203 (1980).

[53] Y. Oh, S. Kim and S. H. Lee, Phys. Rev. C 65, 067901 (2002). 